\begin{tabular}{|c|c|c|c|}
\hline Eiszeitalter und Gegenwart & $\mathbf{5 4}$ & $\begin{array}{c}1-19 \\
8 \text { Abb., } 1 \text { Tab. }\end{array}$ & Hannover 2004 \\
\hline
\end{tabular}

\title{
Rand- und subglaziale Rinnen in den Vorbergen des Süd-Schwarzwaldes bei Bad Säckingen, Hochrhein
}

\author{
Arnfried Becker \& Susanne Angelstein ${ }^{*}$
}

Keywords: subglaziale Rinnen, Randrinnen, Hochrheingebiet, Doppelwall-Riß

Kurzfassung: Das Untersuchungsgebiet liegt auf einem Vorbergsporn des Süd-Schwarzwaldes, der über $2 \mathrm{~km}$ in das Hochrheintal hineinragt. Auf diesem Felsrücken, der vorwiegend aus Gneisen, Graniten und Rotliegend-Klastiten besteht und i.A. nur eine geringmächtige Quartärüberdeckung aufweist, ist ein Talsystem entwickelt, das als subglaziales bzw. randglaziales Rinnensystem interpretiert werden kann. Dafür spricht neben der Lage des Rinnensystems auf einem Hartgesteinsriegel nahe der Ablationsfront eines ehemaligen Gletschers vor allem die Geometrie der Täler mit ihren unausgeglichenen Längsprofilen, ihren trogförmigen Querprofilen mit sehr steilen Talflanken, die teilweise Klippen bilden, ihre lokal begrenzte Verbreitung sowie ein weitgehend fehlendes Einzugsgebiet. Das Entstehungsalter des Rinnensystems wird aufgrund der relativ frischen Geländeformen in das Doppelwall-Riß gestellt. Zu diesem Zeitpunkt lag das Untersuchungsgebiet im Einflussbereich des Rhein- und des Schwarzwaldgletschers. Weiterführende Arbeiten werden vorgeschlagen, u.a. Datierungen des Rinnensystems mit Hilfe von Expositionsaltersbestimmungen.

[Subglacial and lateral channels in the foothills of the southern Black Forest near Bad Säckingen, Hochrhein]

*Anschrift der Verfasser: A. Becker, Sonneggstrasse 57, CH-8006 Zürich, e-mail:

arnfried.becker@bluewin.ch,

S. Angelstein, Mittelberg 3, D-37085 Göttingen, e-mail: Angelstein@gmx.net
Abstract: The area of investigation is on top of a foothill spur of the southern Black Forest that extends southwards into the Rhine valley. The spur is formed from hard rocks, mainly gneisses, granites and Permian clastics, occasionally covered by a thin veneer of Quaternary deposits. The area contains valleys which are regarded as subglacial and lateral drainage channels, respectively. Evidence includes the position of the channel system on top of this hard rock swell close to the termination of a former glacier, the geometry of the valleys, particularly their unequilibrated gradients, their trough-like profiles with very steep valley flanks frequently forming cliffs, their limited extent and the largely missing catchment area. A so-called 'Doppelwall-Riss' age is suggested for the channel system, based mainly on the presence of still fresh-looking geomorphological structures and only moderately weathered rock surfaces. The Doppelwall-Riss belongs to the Riss Complex of the Alpine mid-Pleistocene stratigraphy, which includes the time when the youngest Rhine and Black Forest glaciers reached the area of investigation. Further investigations are proposed, including the dating of the channel system using exposure ages of rock surfaces.

\section{Einleitung}

Der Bergsee nördlich von Bad Säckingen in Deutschland (Abb. 1, 2) war in den vergangenen Jahren mehrmals das Ziel von Untersuchungen, die vom Institut für Geophysik der ETH Zürich im Rahmen eines paläoseismologischen Forschungsprojektes angeregt wurden. Über die Ergebnisse, die den Bergsee direkt betreffen, wurde bereits anderweitig berichtet 

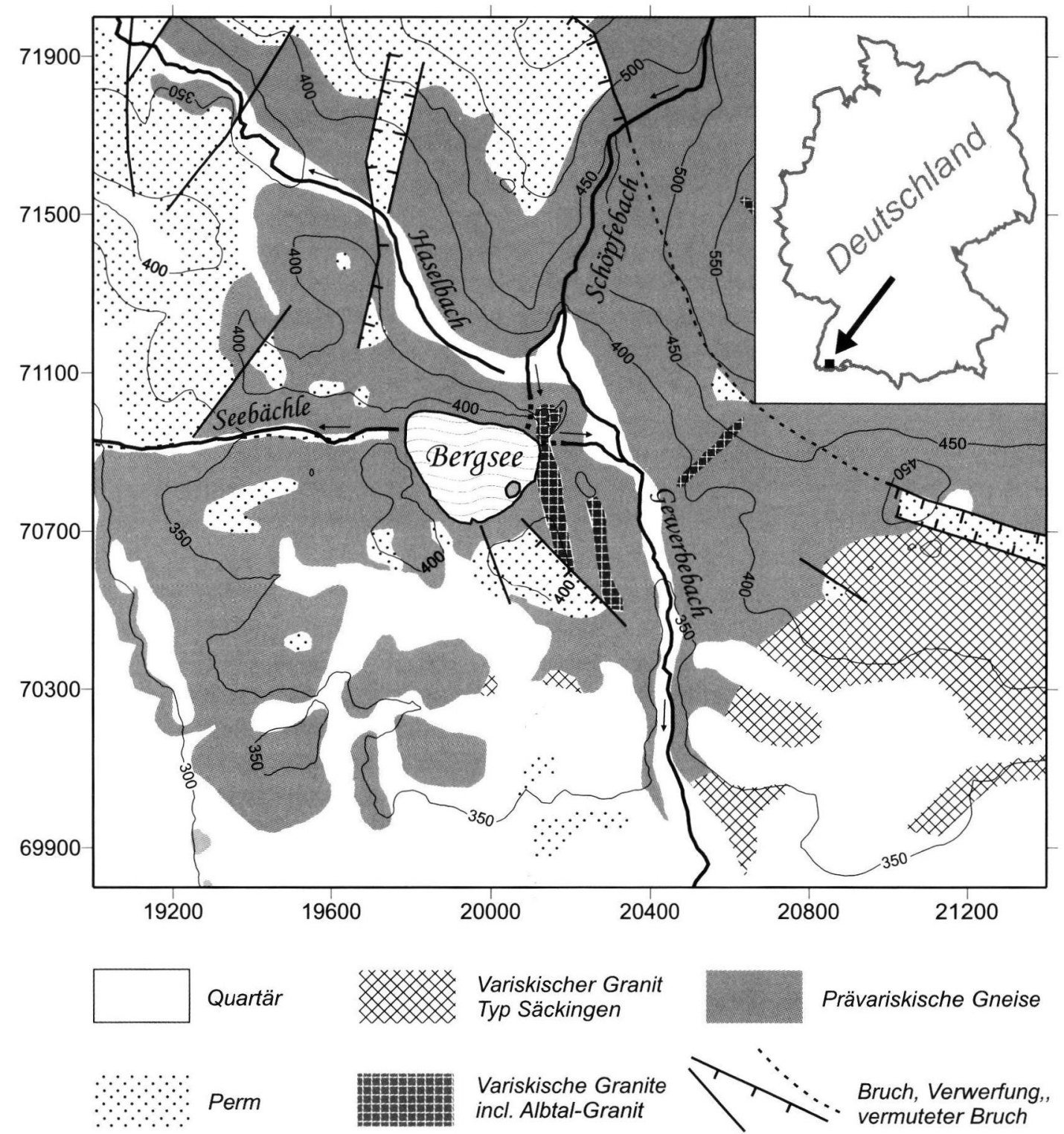

Abb. 1:Geologie und Hydrogeographie des Arbeitsgebietes nördlich von Bad Säckingen in SWDeutschland.

Fig. 1: Geology and drainage of the area of investigation N of Bad Säckingen in SW Germany.

(BeCKer 2003, Becker et al. 2002, 2004). Die Beschäftigung mit dem Bergsee führte zu der Frage, wie dieser See entstanden sein könnte. Geländebegehungen, teilweise zusammen mit Kollegen von den Geographischen Instituten der Universitäten Basel und Zürich, vor allem aber die Kartierung der Geländeformen durch Angelstein (2002), die sie während ihres 2-monatigen Berufspraktikums an der ETH und Universität Zürich durchführte, ergaben wichtige Hinweise zur Entstehung des Bergsees und seiner Umgebung. Darüber hinaus liefer- 

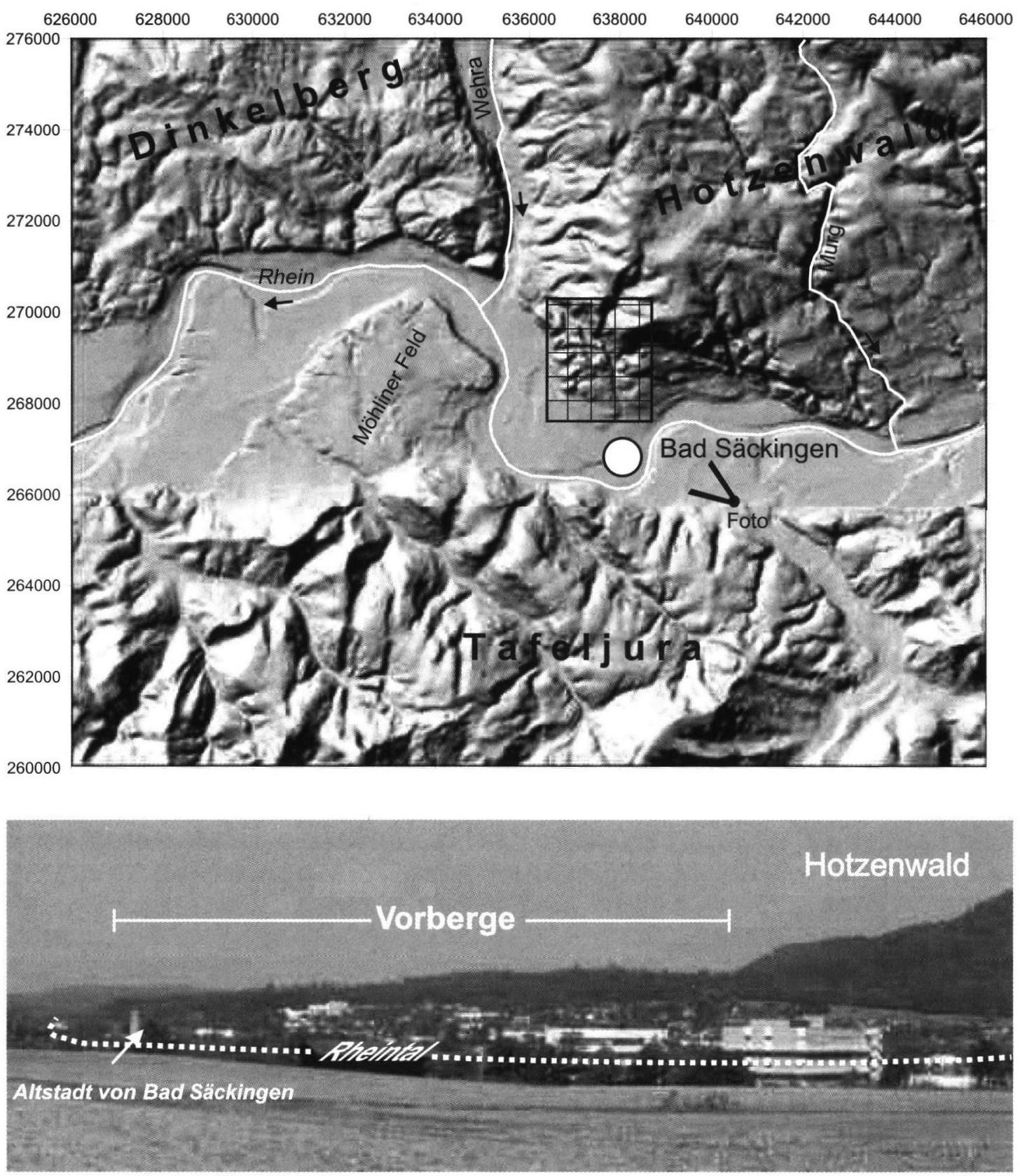

Abb. 2: Digitales Höhenmodell (DHM25 (C) 2003 swisstopo (BA035667)) des Hochrheingebietes um Bad Säckingen mit Lage des Arbeitsgebietes (Kreuzschraffur) sowie eine fotografische Ansicht der SchwarzwaldVorberge von SE (Standort vgl. DHM, Foto: A. Becker, 24.8.2003).

Fig. 2: Digitale Topographic Elevation-Model (DHM25 (C) 2003 swisstopo (BA035667)) of the Hochrhein area around Bad Säckingen showing the location of the area of investigation (squares). A photograph showing the spur of the Black Forest foothills from the SE (position indicated in DHM, photograph: A. Becker, 24.8.2003). 
ten neue Bohrungen, die im nördlich an den Bergsee angrenzenden Haselbachtal im Auftrag der Schluchseewerke AG abgeteuft wurden und deren z.T. noch nicht veröffentlichte Bohrprofile freundlicherweise zur Verfügung gestellt wurden, weitere interessante Einsichten. Diese Ergebnisse und die Daten der Bohrungen und geophysikalischen Untersuchungen aus dem Bergsee führten schließlich zu einem Modell der Morphogenese, das im Folgenden vorgestellt und diskutiert werden soll.

\section{Geographisch-geologischer Überblick}

Das Untersuchungsgebiet liegt in SW-Deutschland am Fuße des Süd-Schwarzwaldes im Gebiet nördlich von Bad Säckingen (Abb. 1, 2). Es wird im Wesentlichen von Gneisen und Graniten des kristallinen Grundgebirges aufgebaut, die teilweise von Rotliegendsedimenten überdeckt sind. Das Untersuchungsgebiet liegt auf einem Vorbergsporn, der sich vom Fuße des Süd-Schwarzwaldes, der hier Hotzenwald genannt wird, über $2 \mathrm{~km}$ in das Hochrheintal hineinzieht. Der Rhein umfließt dieses Hindernis in einem nach $S$ ausweichenden Mäander (Abb. 2). Quartäre Ablagerungen sind im N des Arbeitsgebietes nicht vorhanden oder nur auf die Täler beschränkt (Abb. 3). Im S nimmt ihre Verbreitung rasch zu, wo sie vorwiegend durch teilweise recht mächtigen Löss bzw. Lösslehm vertreten sind (Abb. 3). Im Übergang zum Rheintal und im Rheintal selbst sind fluviatile und glazifluviatile Schotter dominierend, mit Mächtigkeiten, die in Bohrungen z.T. über 30 $m$ erreichen (Sawatzki 1997). Die Schotter werden verschiedenen Eiszeiten zugeschrieben, wobei die jüngsten Schotter des Würm-Komplexes, die so genannten Niederterrassenschotter (Abb. 3), auf das engere Rheintal beschränkt bleiben. Dort sind sie weit verbreitet und werden nur lokal von jüngeren Schwemmfächern oder von Hangschutt randlich überlagert.
Hochterrassenschotter, die dem Riß-Komplex zugewiesen werden (GeYer et al. 2003, VerDERBER 1992, 2003), stehen im SE und im W des Arbeitsgebietes an (Beising 2003, SAWATZKI 1997). Im SE des Arbeitsgebietes wird ein kleines Vorkommen von SaWATZKi (1997) als rißeiszeitliche Grundmoräne angesprochen. Die ältesten Quartärablagerungen befinden sich in einem isolierten Vorkommen im NW des Arbeitsgebietes (Abb. 3). Dieses Schottervorkommen des Duttenbergs wird als Relikt der Höheren (Älteren) Deckenschotter im Gebiet von Bad Säckingen angesehen (Geyer et al. 2003, Verderber 1992, 2003).

Hydrogeographisch wird das Arbeitsgebiet vom Schöpfebach-Gewerbebach-System, dem Haselbach und dem Seebächle, dem natürlichen Überlauf des Bergsees, bestimmt (Abb. 1). Eingebettet in einer flachhügeligen Landschaft liegt der Bergsee. Er besitzt keinen natürlichen Oberflächenzufluss, sondern wird nur durch Grundwasserzustrom und Oberflächenzufluss von den umgebenden Hängen gespeist. Der heutige Zufluss aus dem Schöpfebach über einen Kanal und in einem Stollen durch den Scheffelfels besteht seit 1804/05. Er dient der Sicherung und Regulierung des Wasserstands im See, der auch heute noch als Wasserreservoir für die Industrie in Bad Säckingen genutzt wird. Durch mehrmalige Erhöhung des Überlaufniveaus zum Seebächle wurde der Seespiegel zwischen 1804 und 1907 um bis zu $6 \mathrm{~m}$ über das natürliche Niveau angehoben (MüLleR 1993). Dadurch und vor allem auch durch die Verschmutzung des künstlichen Zubringers mit ungereinigten Abwässern besonders in den 1960er und 1970er Jahren veränderte der See in den vergangenen 200 Jahren seinen Charakter vollständig von einem ursprünglich kleinen dystrophen Gewässer (ursprünglicher Name „Schwarzsee“) zu einem $335 \mathrm{~m}$ x $250 \mathrm{~m}$ und bis zu $12 \mathrm{~m}$ tiefen eutrophen See (Wüthrich 2003). Wie die bisher in Bohrungen erschlossenen, über $20 \mathrm{~m}$ mächtigen Sedimente des 


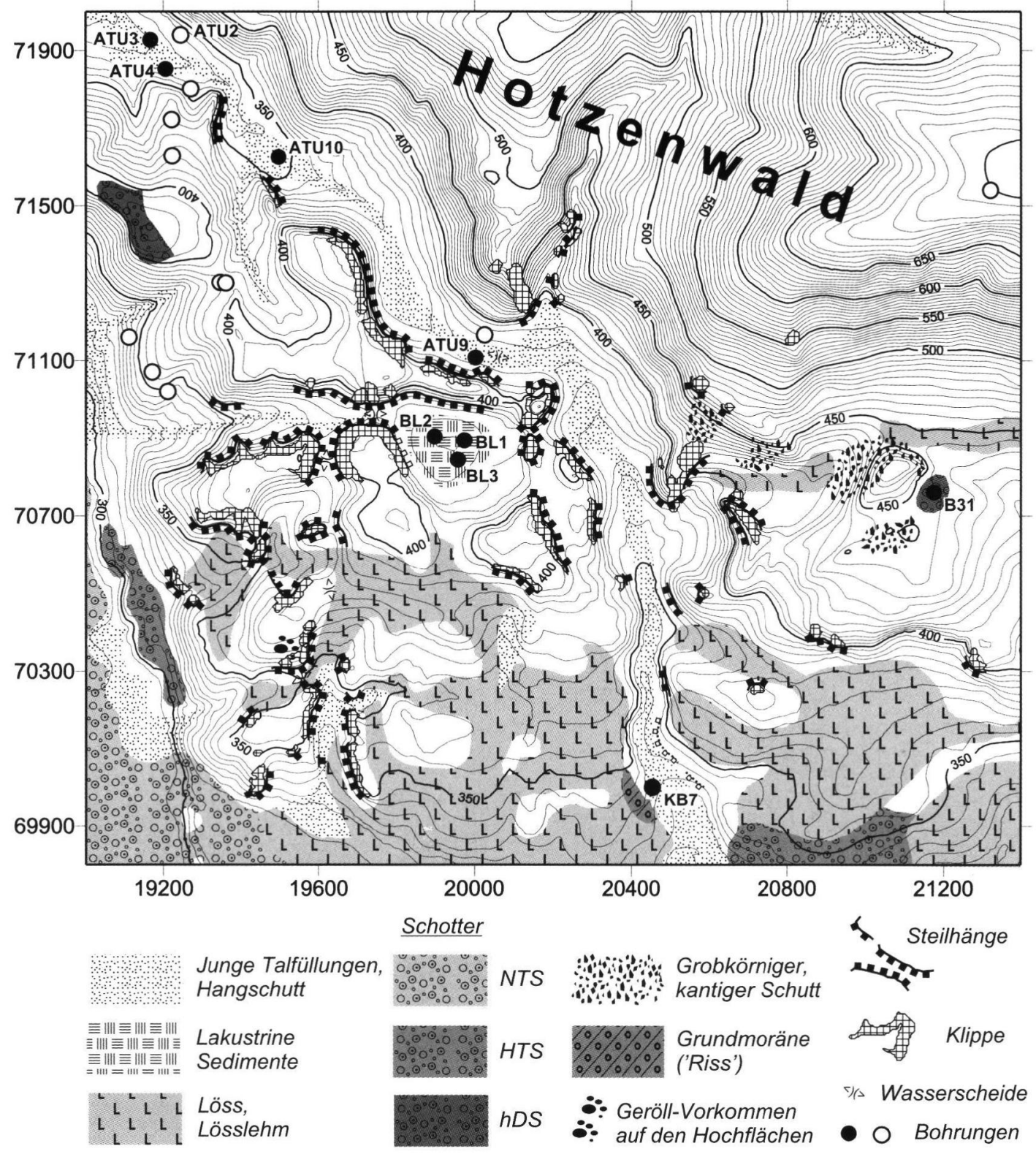

Abb. 3: Verbreitung des Quartärs, Geomorphologie und Lage der Bohrungen im Arbeitsgebiet. NTS : Niederterrassenschotter, HTS : Hochterrassenschotter, hDS : höhere Deckenschotter. Bohrpunkte in schwarz und mit Bezeichnungen werden im Text erwähnt.

Fig. 3: Distribution of Quaternary deposits, geomorphology and drill sites in the area of investigation. Gravels of the NTS : Lower Terrace, HTS : Higher Terrace, and hDS : upper Sheet Gravel Deposits. Drill sites in black and with signatures are mentioned in the text. 


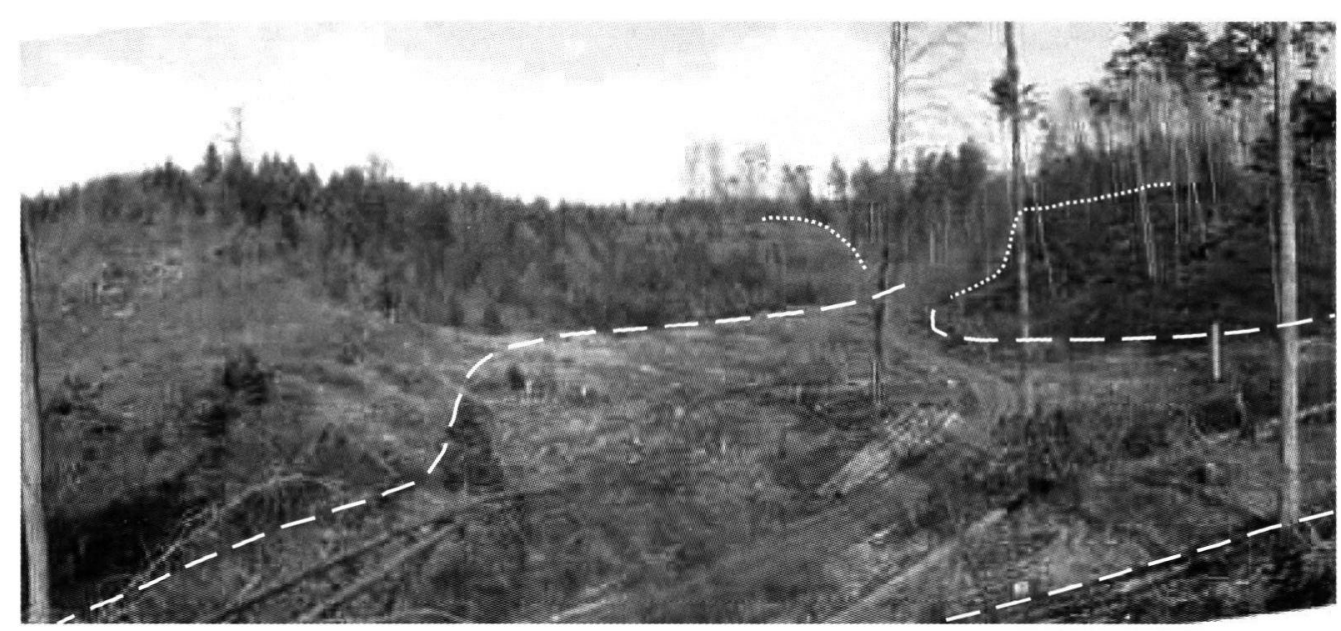

Abb.4: Tal südwestlich des Bergsees mit typischer Morphologie. Der ungefähre Verlauf des Talrandes ist durch gestrichelte, die Hangtopographie durch punktierte Linien markiert. Ein intermittierendes Rinnsal entwässert das Tal. Foto: A. Becker, 19.1.2003.

Fig. 4: Photograph of a valley SW of Lake Bergsee with a characteristic topography. The approximate extent of the valley margin has been indicated by hatched lines, the topography of the slopes by dotted lines. A small intermitting stream drains the valley. Photograph: A. Becker, 19.1.2003

Bergsees belegen, läßt sich seine Entwicklung anhand von Radiokohlenstoffdatierungen und palynostratigraphischen Untersuchungen mindestens bis ins Denekamp-Interstadial vor ca. 29‘000 Jahren vor heute zurückverfolgen (BECKER et al. 2002, BECKER 2003).

\section{Problemstellung}

Die vermeintlich isolierte Lage des Bergsees in einem Höhenzug deutlich über dem Niveau des Rheintals, zudem ohne natürlichen Oberflächenzufluss aber mit mächtiger Sedimentfüllung, führten uns zu der Frage, wie dieser See entstanden sein könnte. Einige Annahmen zur Seegenese konnten aufgrund der regionalen Geologie sofort ausgeschlossen werden. Dazu gehört Subrosion, die in den Kristallingesteinen des Untersuchungsgebietes nicht zu erwarten ist. Für die Entstehung des Bergsees infolge eines kosmischen Impakts oder einer phreatomagmatischen Explosion
(Maar) gibt es keine Evidenz. Auch diese Annahmen können daher ausgeschlossen werden. Es bleibt die Möglichkeit einer tektonischen Entstehung durch Absenkungen entlang von Extensionsstrukturen. Obgleich im näheren Umfeld des Bergsees verschiedentlich auf die Existenz neotektonischer Absenkungen entlang bedeutender Bruchsysteme hingewiesen wurde, z.B. der Wehratal-Zeininger Bruchzone unmittelbar westlich des Untersuchungsgebietes oder der Vorwaldstörung (Haldimann et al. 1984, Trefzger 1965), wurden solche Hinweise zumeist kontrovers diskutiert und nicht allgemein anerkannt, teilweise auch widerlegt (GEYER et al. 2003, Verderber 1992, 2003). Für die nähere Umgebung des Bergsees sind keine Bruchsysteme mit jungen tektonischen Absenkungen bekannt, die zur Entstehung des Bergsees geführt haben könnten. Es bleiben als Erklärung für die Prägung der Landschaft nördlich von Bad Säckingen mit dem Bergsee in ihrer Mitte nur glazifluviatile oder glaziale Prozesse übrig, ähnlich denen, die im Schwarzwald, den 
benachbarten Vogesen, den Alpen und ihrem Vorland vielfach zur Entstehung von Seen nach dem Abschmelzen der Gletscher geführt haben. Im Folgenden wird untersucht, welche glazifluviatilen Prozesse das gewesen sein könnten und wann sie wirksam waren.

\section{Geländebeobachtungen}

\subsection{Geomorphologie}

Der Höhenrücken, der vom Rand des Hotzenwaldes als Härtlingssporn in das Hochrheintal hineinzieht, wird von Tälern durchzogen, die einige Besonderheiten aufweisen. In einem dieser Täler liegt der Bergsee (Abb. 1, 3). Am deutlichsten treten diese Täler in der Umgebung des Bergsees und südwestlich davon hervor, wohingegen sie im S und SE kaum noch in Erscheinung treten, teilweise bedingt durch eine zunehmend mächtiger werdende Überdeckung mit quartären Sedimenten (Beising 2003), insbesondere Löss und Lösslehm. Im Hotzenwald fehlen sie völlig. Im Gegensatz zu den Hotzenwaldtälern, Beispiel Schöpfebach (Abb. 1), die kerbförmig eingeschnitten, teilweise von Klippen gesäumt sind und meistens von einem Fliessgewässer durchzogen werden, haben die Täler in den Vorbergen eine breite Talsohle, werden meistens nur - wenn überhaupt - durch ein intermittierendes Rinnsal durchzogen und haben oft sehr steile Talflanken, die mitunter in Klippen übergehen mit Höhen von teilweise mehr als $20 \mathrm{~m}$. Ein typisches Beispiel eines solchen Tales ist in Abbildung 4 zu sehen, aufgenommen ca. $500 \mathrm{~m}$ südwestlich des Bergsees $\left({ }^{34} 19500 / 5270530\right.$ mit Blick nach NNE). Es zeigt einen weiten Talboden mit einem kleinen intermittierenden Bach, relativ flachen Talflanken im W (links) und steilen, teilweise klippenartigen im E (rechts). Die Querschnittsprofile der Täler sind zumeist symmetrisch oder nur schwach asymmetrisch. Eine Ausnahme ist das Haselbachtal (Abb.1, 3). Die Querprofile A - C
(Abb. 5) zeigen deutlich, dass die südwestlichen Talflanken viel schroffer und steiler aufragen als die nordöstlichen Talflanken, die den Abfall des Hotzenwaldes markieren. Im Profil D geht dieser Gegensatz zwischen den beiden Talseiten aber bereits verloren, und es kann hier wieder von einem weitgehend symmetrischen Talquerschnitt gesprochen werden. Im Gegensatz zu den anderen zuvor erwähnten Tälern besitzt das Haselbachtal zumindest einen persistierenden Bach, dessen Wasserführung und dessen Einzugsgebiet allerdings im auffallenden Kontrast zu der Größe des Tales stehen. In den Tälern, in denen Fels an oder nahe der Talsohle ansteht, sind in den Längsprofilen Wasserscheiden ausgebildet, besonders gut zu erkennen östlich und westlich des Bergsees (Abb. 1, 3). Die Talsohlen zeigen demnach kein für Oberflächengewässer typisches einseitig gerichtetes Gefälle. Eigenartig ist auch die regionale Verteilung der Täler. Sie sind besonders deutlich um den Bergsee und südwestlich davon ausgebildet, hingegen weit undeutlicher östlich des Bergsees. Darüber hinaus verzweigen sich die Täler und ändern in einigen Fällen abrupt ihre Richtungen (Abb. 3). Ein natürliches Einzugsgebiet für dieses Talsystem ist nicht ersichtlich, legt man die heutige Topographie zu Grunde. Auch ist ein Zusammenhang zwischen dem Vorkommen der Klippen und der lokalen Lithologie nicht offensichtlich. Zwar sind die meisten Klippen, entsprechend der Verbreitung dieser Gesteine im Untersuchungsgebiet, in den prävariskischen Gneisen angelegt. Es gibt sie aber auch in den klastischen Rotliegendsedimenten, beispielsweise SSE des Bergsees, oder im variskischen Granit vom Typ Säckingen, dort allerdings weniger gut ausgeprägt. Nur östlich des Bergsees scheinen die harten, gangartigen Vorkommen des Albtalgranits die Lage einiger Klippen bestimmt zu haben (Abb.1, 3). 

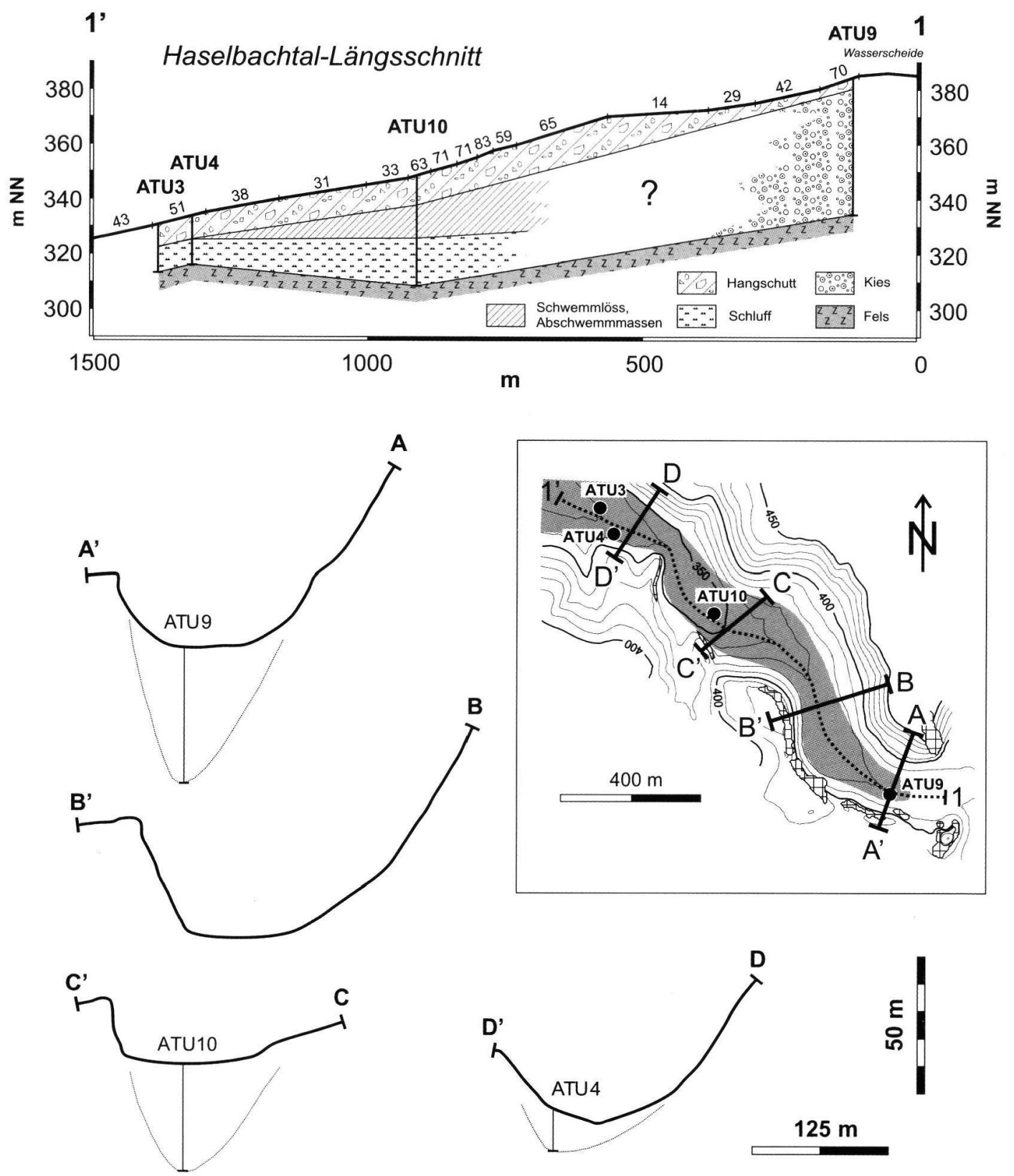

Abb. 5: Längsschnitt durch das Haselbachtal mit Angaben des Gefälles (in \%o), mit den in die Profillinie projizierten Bohrpunkten und Angaben zur Sedimentfüllung. Die Lage des Längsschnitts und der vier morphologischen Querprofile zeigt die Lageskizze.

Fig. 5: Section along the centre of the Haselbach valley, with stream gradients (shown as \%o), boreholes projected onto the section line, and sediment thicknesses. The position of the long section and the four crosssections is indicated in the sketch map. 


\subsection{Bohrungen}

Die Lage der Bohrpunkte im Untersuchungsgebiet ist der Abbildung 3 zu entnehmen. Die Bohrungen, die ein mächtigeres Quartär aufweisen, sind benannt und mit einem schwarzen Punkt markiert. Vereinfachte lithologische Profile nach Becker et al. (2002), BeCKer (2003) und SAWATZKi (1997) sowie noch unveröffentlichten Daten der Schluchseewerke AG sind der Abbildung $6 \mathrm{zu}$ entnehmen. Besonders interessant sind die Kernbohrungen aus dem Haselbachtal, die z.T. ganz außergewöhnliche Quartärmächtigkeiten aufweisen. Die Bohrung ATU9, nur $150 \mathrm{~m}$ Luftlinie nördlich des Bergsees im oberen Haselbachtal gelegen, weist $50 \mathrm{~m}$ grobkörnige, schwach gerundete bis kantige Kiese und Schotter auf, wobei nur die obersten $4 \mathrm{~m}$ als rezenter oder subrezenter Hangschutt angesehen werden können. Die Felsbasis wurde in 334,5 m NN angetroffen. In der Bohrung ATU10 ca. $700 \mathrm{~m}$ talabwärts ist das Quartär noch immer knapp $40 \mathrm{~m}$ mächtig (SAWATZKI 1997). In den obersten $10 \mathrm{~m}$ besteht es aus rotem bis hellbraunem, sandig-steinigem tonigen Hangschutt. Darunter folgen Abschwemmmassen bestehend aus roten, braunen, teilweise dunkelgrauen organischen, sandigen, teilweise steinigen Tonen und Schwemmlöss mit einer Mächtigkeit von $12 \mathrm{~m}$. An der Basis wurden $17 \mathrm{~m}$ feingeschichtete, feinsandige, graue, grüngraue und rote Schluffe angetroffen, bevor die Felsoberfläche in einer Tiefe von 308,6 m NN erreicht wurde. In den Bohrungen ATU4 und ATU3 bleiben die Quartärmächtigkeiten unter $20 \mathrm{~m}$, wobei die obersten ca. $8 \mathrm{~m}$ (Abb. 6) als Hangschutt angesprochen werden können. Darunter folgen rote und graue, feingeschichtete, feinsandige, z.T. organische Schluffe, die grosse Ähnlichkeit mit den Schluffen in der Bohrung ATU10 haben. Die Felssohle wird in den Bohrungen ATU4 und ATU3 in 316,3 m bzw. 313,4 m angetroffen. Sie liegt damit 5 - $8 \mathrm{~m}$ über der Felssohle in ATU10 (Abb. 5,
6). Damit deutet sich für das Haselbachtal eine Übertiefung der Fels-Talsohle an, was im Falle von Oberflächengerinnen nur unter besonderen Bedingungen erwartet werde kann (Kolkbildung). Auch das rezente Gefälle des Haselbachtals ist nicht ausgeglichen, sondern zeigt Verebnungen und Steilstrecken mit Gradienten, die zwischen $14 \%$ und $83 \%$ variieren (Abb. 5). Interessant ist auch, dass die heutige Wasserscheide zwischen dem Haselbach- und dem Schöpfebachtal nicht, wie vielleicht vermutet werden konnte, auf Fels oder geringmächtigem Hangschutt liegt, sondern offensichtlich auf einer Schotterauffüllung, die bis zu $50 \mathrm{~m}$ mächtig ist. Unklar bleibt allerdings die Verzahnung zwischen den Kiesen und Schottern der Bohrung ATU9 mit den tieferen Sedimenten der Bohrung ATU10, insbesondere die Frage, ob die Schluffe an der Basis der Bohrungen ATU10, ATU3 und ATU4 lateral die Kiese und Schotter in ATU9 vertreten (Abb. 5).

Im Bergsee wurden an drei Orten fünf Kernbohrungen bis zu einer maximalen Tiefe von knapp $21 \mathrm{~m}$ abgeteuft (Abb. 3, 6). Die obersten spätpleistozänen und holozänen Seeablagerungen sind fast ausschließlich organisch und bestehen aus Gyttja, Detritus-Gyttja und organischem Detritus (Becker 2003). An der Basis dieser Sedimente fällt die Laacher See Tephra als weiße bis hellgrüne schluffige Einschaltung auf. Unter den organischen Sedimenten folgen hellbraune und grünliche tonige Schluffe und Schluffe, die nahe der Basis der tiefsten Bohrung (BL3.1) wieder leicht organisch werden. Hier wurde eine Probe mit der Radiokohlenstoffmethode auf 29'110 \pm 280 uncal. BP datiert (BeCKer et al. 2002, BeCKer 2003), womit dieser Abschnitt in das Denekamp-Interstadial gestellt werden kann. Diese Einstufung wird auch durch Pollenanalysen bestätigt, die ein Wiederauftreten von Baumpollen (Betula, Pinus, Juniperus) belegen (Becker 2003). Die Endteufe der Bohrung BL3.1 liegt bei $351,9 \mathrm{~m}$ $\mathrm{NN}$, ohne jedoch die Felsoberfläche erreicht zu 

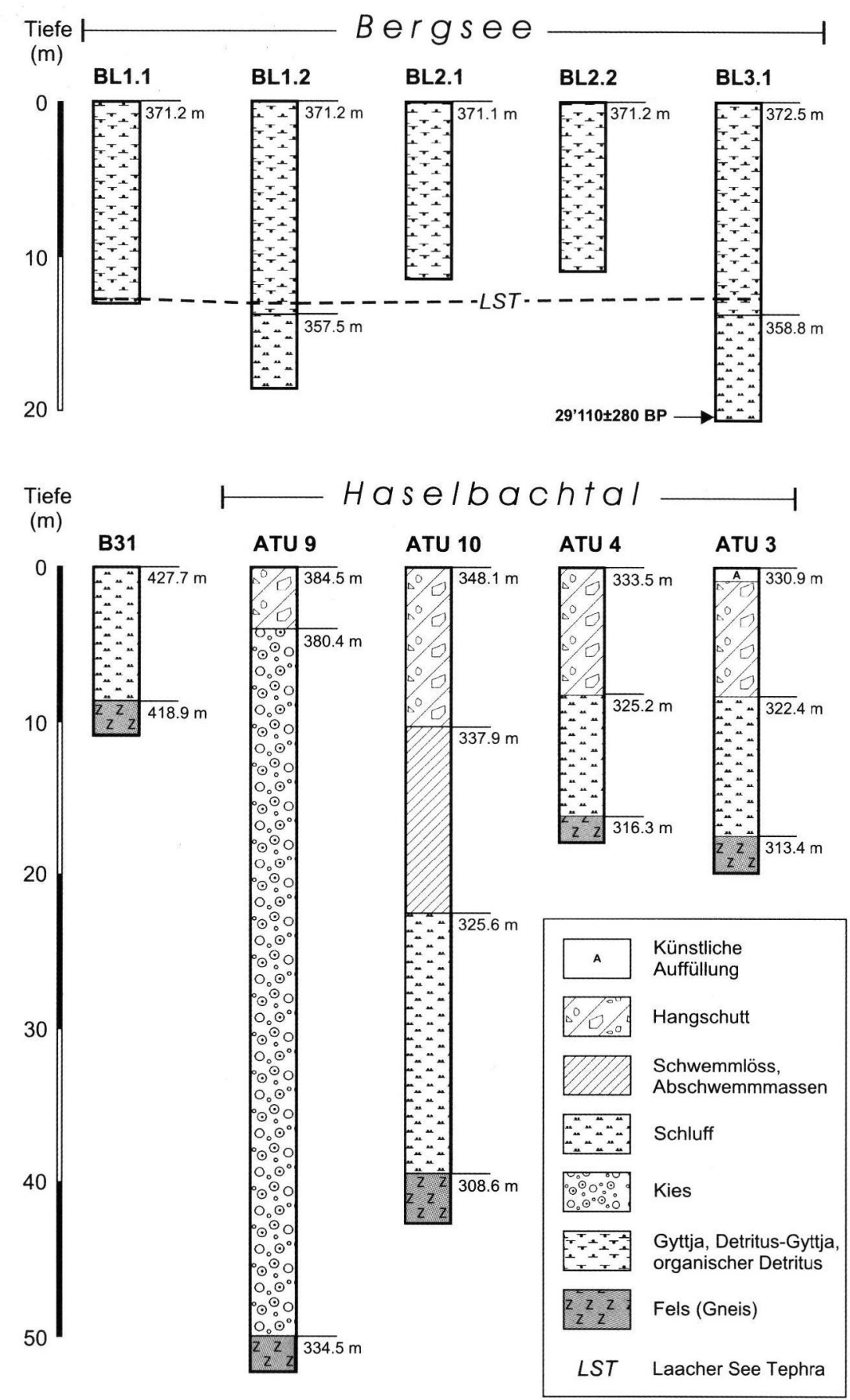

Abb. 6: Vereinfachte lithologische Profile einiger Bohrungen im Gebiet des Bergsees mit mächtigem Quartär

Fig. 6: Simplified lithologs of boreholes containing thick Quaternary deposits in the Bergsee region. 
haben. Nach neuesten, noch unpublizierten geophysikalischen Untersuchungen könnte das Seebecken noch deutlich tiefer sein und insgesamt eine Sedimentfüllung von $45 \pm 15 \mathrm{~m}$ erreichen (mdl. Mitt. F. Marillier, Lausanne). Dieser Wert liegt somit sehr nahe an dem Wert für die Sedimentmächtigkeit in der Bohrung ATU9 im Haselbachtal. Somit deutet sich auch für das Tal, in dem heute der Bergsee liegt, eine Übertiefung der Felssohle ähnlich der im $\mathrm{Ha}$ selbachtal an.

Eine weitere Bohrung, die mächtigeres Quartär zeigt, ist B31 (Abb. 3, 6) im E des Untersuchungsgebietes $\left({ }^{34} 21174 /{ }^{52} 70760\right)$. Hier besteht das Quartär aus knapp $8 \mathrm{~m}$ mächtigen, rotbraunen tonigen und kiesigen Schluffen. Die Herkunft der gröberen Komponenten kann teilweise als alpin eingestuft werden, meistens stammen sie aber aus dem Schwarzwald (SaWATZKI, 1997). Alle anderen Bohrungen im Untersuchungsgebiet (Abb. 3) zeigen nur geringmächtiges Quartär, so auch die Kernbohrung KB7 ( $\left.{ }^{34} 20355 / 5^{2} 70000\right)$ im unteren Schöpfebachtal, wo tonige bis sandige Kiese nur $2 \mathrm{~m}$ mächtig werden, bevor die Felsoberfläche erreicht wird (SAWATZKI 1997).

\section{Diskussion und Interpretation}

\subsection{Subglaziale Rinnen und Randrinnen}

Es werden zwei Arten subglazialer Rinnen unterschieden: Röthlisberger Channels (RChannels), die sich an der Gletscherbasis in den überlagernden Gletscher einschneiden und die Nye-Channels (N-Channels), die sich von der Gletscherbasis in das unterlagernde Gestein einschneiden. Nach dem Abschmelzen des Gletschers sind nur noch die N-Channels zu sehen. Diese N-Channels sind Erosionsformen, die durch sedimentbeladene Schmelzwasserströme entstehen, die aufgrund der Gletschereisüberdeckung unter teilweise sehr hohem hydrostatischen Druck stehen und turbulent mit hohen Geschwindigkeiten in röhrenartigen Rinnen fließen (Benn \& Evans 1998, MenzIES 1996). Grobschluff- und sandbeladene Schmelzwasserströme hoher Geschwindigkeit sind besonders korrasiv, deren Wirkung bei turbulentem Fließen noch durch Kavitation an Engstellen des Rinnensystems mit besonders hohen Fließgeschwindigkeiten verstärkt werden kann (Benn \& Evans 1998, Sudgen \& John 1976). N-Channels bilden sich vorzugsweise auf Hartgesteinsunterlagen - so genannten H-beds, wie z.B. Fels (Ha-beds), gefrorenem Schutt (Hb-beds) oder Lockergestein mit kleinem Durchlässigkeitsbeiwert (Hc-beds) (Menzies 1996) - an der Basis temperierter Gletscher nahe der Ablationsfront, wo große Schmelzwassermengen anfallen (Воотн \& Hallet 1993, Embleton \& King 1975, Sudgen \& John 1976). Solche subglazialen Rinnen können ganz unterschiedliche Dimensionen erreichen, von einigen Dezimetern Breite und wenigen Metern Länge bis zu mehreren Kilometern Länge, $1000 \mathrm{~m}$ Breite und mehreren Hektometern Tiefe. Sehr große subglaziale Rinnen werden auch Tunneltäler genannt (BenN \& Evans 1998). Subglaziale Rinnen setzen plötzlich ein, schneiden sich mitunter tief in den Fels ein, verzweigen sich und verschwinden oft unvermittelt. Dieses Phänomen ist damit zu erklären, dass das Schmelzwasser teilweise im Gletschereis in den R-Channels fließt und sich nur örtlich an der Basis des Gletschers in den anstehenden Fels als N-Channel einschneidet. Nach dem Abschmelzen des Gletschers sind dann nur noch diese N-Channels an der ehemaligen Basis des Gletschers erhalten mit einer unausgeglichenen, z.T. fluktuierenden Gefällekurve und einem für die Breite und Tiefe der Täler ungewöhnlich kleinen Einzugsgebiet (Menzies 1996). In Bezug auf die heutige Topographie können die subglazialen Rinnen sogar gegen das Gefälle bergauf verlaufen. Da die Entstehung dieser subglazialen Rinnen durch den hydrauli- 

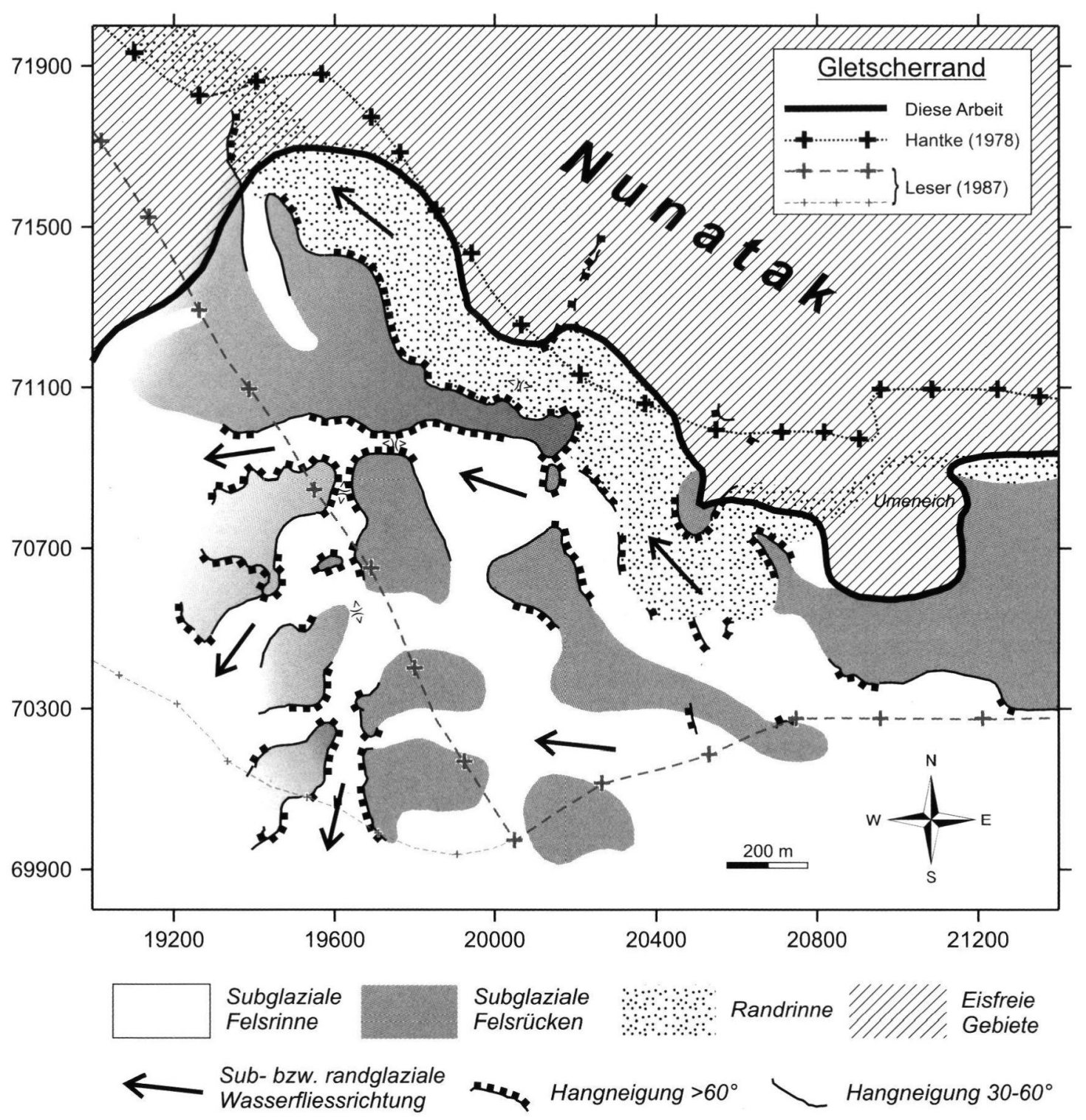

Subglaziale

Felsrücken

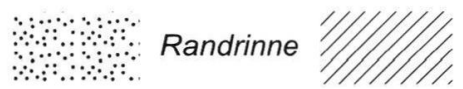

Eisfreie

Gebiete

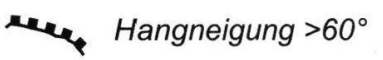

Hangneigung $30-60^{\circ}$

Abb. 7: Lage der subglazialen Felsrinnen und der Randrinne im Bergseegebiet. Im Gebiet südlich des Bergsees zeichnet sich das Rinnensystem aufgrund der Quartärauflage nur undeutlich ab. „Riß-Eiszeitliche” maximale Gletscherstände (große Kreuze) nach Hantke (1978) und LeSER (1987) sowie eines schwächeren rißzeitlichen Gletschervorstoßes (kleine Kreuze) nach LEsER (1987) sind markiert.

Fig. 7: Location of the subglacial channels and the lateral drainage channel in the Bergsee region. South of Bergsee the channel system is hidden by thick Quaternary deposits. The maximum stand of the Rhine glacier during the „Riss ice-age“ (large crosses) after HantKe (1978) and Leser (1987) and a much weaker glacier advance (small crosses) after LeSER (1987) are indicated in the map. 
schen Gradienten bestimmt wird, der vor allem durch die Gletscheroberfläche dominiert, von der Topographie der Gletschersohle aber nur beeinflusst wird, kann sich ein Rinnensystem an der Basis des Gletschers ausbilden, in dem das Schmelzwasser vom Bereich größter Eisdicke zum Gletscherrand fließt (Воотн \& Hallet 1993, Menzies 1996, Sudgen \& John 1976). Nur in den Fällen, wo die subglazialen Rinnen nicht vollständig wassergefüllt sind, folgen sie dem Gefälle der subglazialen Geländeoberfläche (Benn \& Evans 1998).

Die Tabelle 1 listet Charakteristika subglazialer Rinnen (N-Channels) nach BENN \& Evans (1998), Embleton \& King (1975) und MenZIES (1996) auf und stellt die Beobachtungen aus dem Bergseegebiet gegenüber. Deutlich wird, dass die meisten Kriterien zur Erkennung subglazialer Rinnen auch im Bergseegebiet erfüllt werden. Das trifft vor allem auf die Abmessungen, die Gesteinsunterlage sowie in den meisten Fällen auch auf die Lage der Rinnen in Bezug auf die Hartgesteinsschwelle und auf die charakteristischen Geländeformen zu. Glatte und gerundete Felsoberflächen wurden nur lokal beobachtet, z.B. am Scheffelfels im AlbtalGranit sowie den Gneisen entlang der östlichen Talflanke des Tales im SW des Untersuchungsgebietes ( ${ }^{34} 19600 /{ }^{5270100)}$. Kleinmaßstäbliche p-Formen (DAHL 1965) wurden aber nirgendwo beobachtet. Möglicherweise wurden diese p-Formen, wie z.B. Sichelwannen, Spindelmarken, Muschelbrüche, nach ihrer Entstehung durch Verwitterungsprozesse zerstört oder primär gar nicht gebildet (vgl. Bоотн \& Hallet 1993). Die Lage der subglazialen Rinnen und die generelle Schmelzwasser-Fließrichtung ist in Abbildung 7 wiedergeben.

Die Lage des Haselbachtals am Fuße des Hotzenwaldes, vor allem die ausgeprägte Asymmetrie der Talquerprofile, die ohne Sedimentverfüllung noch deutlicher hervorträte, lassen auf eine andere Entstehung dieses Tales im Vergleich zu den subglazialen Rinnen weiter im S schließen.
Sehr wahrscheinlich handelt es sich hierbei um eine so genannte Randrinne, die sich am Rande des Gletschereises bildete. Randrinnen sind die Sammler des Schmelzwassers, das sich auf dem Gletscher gebildet hat und randlich abfließt, oder von Wasser, das dem Gletscherrand aus den eisfreien Gebieten zuströmt. In diesen Randrinnen fließt das Wasser entweder direkt am Gletscherrand oberflächlich ab, kann aber auch infolge von Hindernissen vom Gletscherrand in das Nebengestein abgedrängt oder auch randlich subglazial abgeführt werden (EMBLETON \& KING 1975). Berücksichtigt man die Übertiefung der Felssohle des Haselbachtals (Abb. 5), dann muss sich diese Randrinne ebenfalls zumindest zeitweilig unter dem Eis gebildet haben. Diese Randrinne zieht aus dem Haselbachtal in das Schöpfebachtal zwischen den Koten 400 und 350 m NN, wo die ehemalige Sedimentverfüllung der Randrinne teilweise durch den Schöpfebach ausgeräumt wurde und ein relativ weites Tal mit teilweise asymmetrischem Talquerschnitt entstand. Die weitere Fortsetzung der Randrinne ist nicht klar. Wahrscheinlich zieht sie nach $\mathrm{E}$ zwischen den Hotzenwaldfuß und die teilweise mit Periglazialschutt überdeckte Felskuppe von Umeneich (Abb. 7), wo die Randrinne vermutlich oberflächlich verlief, bevor sie östlich von Umeneich wieder den ehemaligen Eisrand erreichte. Sicherlich folgte die Randrinne aber nicht dem relativ engen, nur mit geringmächtigem Quartär verfüllten oberen Gewerbebachtal unterhalb Kote 350 m NN (Abb. 1, 3, 7). Dieser Teil des Schöpfebach-Gewerbebach-Systems ist wahrscheinlich erst nach der Bildung der Randrinne entstanden.

Aus der Lage der Randrinne und der Verbreitung der subglazialen Felsrinnen kann auf die Verbreitung des Gletschers und die ungefähre Höhe des Gletscherrandes am Fuße des Hotzenwaldes geschlossen werden. Nördlich des Bergsees verlief der Gletscherrand ungefähr bei $400 \mathrm{~m} \mathrm{NN}$, fiel auf ca. $340 \mathrm{~m} \mathrm{NN}$ nach W ab, 
Tab.: 1 Subglaziale Rinnen (Benn \& Evans 1998, Embleton \& King 1975, Menzies 1996) im

Vergleich mit den Tälern im Bergseegebiet .

Tab.: 1 Characteristics of subglacial channels (Benn \& Evans 1998, Embleton \& King 1975, Menzies 1996) compared with the valleys seen in the Bergsee area.

\begin{tabular}{|c|c|}
\hline Subglaziale Rinnen vom Typ N-Channels & Täler im Bergseegebiet \\
\hline $\begin{array}{l}\text { Dimension: } \\
\text { wenige Meter bis einige Kilometer Länge, } \\
\text { bis zu } 1000 \mathrm{~m} \text { breit und einige } 100 \mathrm{~m} \text { tief }\end{array}$ & $\begin{array}{l}\text { Einige } 100 \mathrm{~m} \text { lang und bis zu } 200 \mathrm{~m} \text { breit, } \\
\text { z.T. einige Dekameter tief. }\end{array}$ \\
\hline $\begin{array}{l}\text { Unterlage: } \\
\text { Vor allem auf Hartgestein (Ha-beds) oder } \\
\text { gefrorenem Lockergestein (Hb-beds) }\end{array}$ & Gneise, Granite, Rotliegend-Klastite \\
\hline 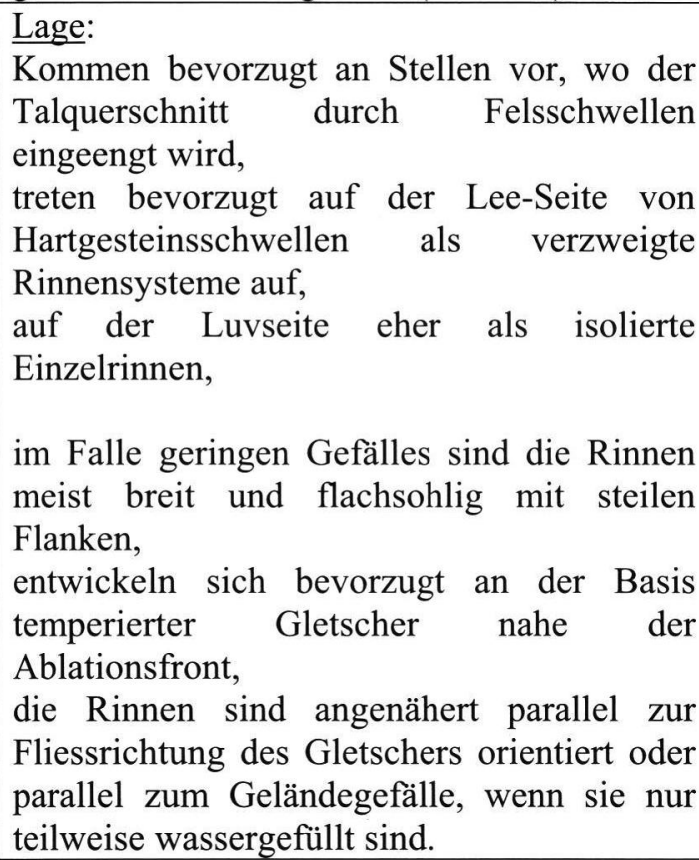 & $\begin{array}{l}\text { Das Bergseegebiet ist Teil einer } \\
\text { Felsschwelle, die vom Schwarzwaldfuß über } \\
2 \mathrm{~km} \text { in das Rheintal hineinreicht. } \\
\text { Im W der Vorberg - Felsschwelle sind die } \\
\text { Täler verzweigt und am deutlichsten } \\
\text { ausgeprägt, } \\
\text { im E und SE kaum mehr zu erkennen und } \\
\text { teilweise durch jüngere Ablagerungen } \\
\text { verdeckt. } \\
\text { Talsohlen sind oft breit und flach (aber nicht } \\
\text { immer auf Fels), die Talflanken oft } \\
\text { klippenförmig. } \\
\text { Das Untersuchungsgebiet liegt nur 300-500 } \\
\text { m NN und damit sicherlich nahe der } \\
\text { Ablationsfront eines ehemaligen Gletschers. } \\
\text { Die Rinnen sind NW-SE, E-W, SW-NE und } \\
\text { N-S orientiert, der ehemalige Gletscher floss } \\
\text { ungefähr von E nach W. }\end{array}$ \\
\hline $\begin{array}{l}\text { Charakteristika: } \\
\text { Abrupte Änderung der Rinnenrichtung, } \\
\text { Rinnen verschneiden sich und enden abrupt } \\
\text { und zeigen fluktuierende Rinnengefälle. } \\
\text { Das Rinnennetz hat eine begrenzte } \\
\text { Verbreitung und } \\
\text { ein Einzugsgebiet fehlt weitgehend. } \\
\text { Die Felsoberflächen sind glatt geschliffen } \\
\text { und abgerundet. }\end{array}$ & $\begin{array}{l}\text { Teilweise Abzweigungen unter } 90^{\circ} \text {. } \\
\text { Rinnen beginnen und enden abrupt. } \\
\text { Lokale Wasserscheiden vorhanden. } \\
\text { Das Rinnennetz ist nur auf die Umgebung } \\
\text { des Bergsees beschränkt und } \\
\text { hat kein bedeutendes Einzugsebiet. } \\
\text { Glatte Felsoberflächen sind selten zu sehen, } \\
\text { am deutlichsten noch am Bergsee } \\
\text { (Scheffelfels) und dem Tal im SW des } \\
\text { Untersuchungsgebietes. }\end{array}$ \\
\hline
\end{tabular}


wo der Gletscherrand aufgrund der Verbreitung der subglazialen Rinnen evtl. weiter südlich verlief, und stieg ungefähr auf $450 \mathrm{~m} \mathrm{NN}$ am Ostrand des Untersuchungsgebietes an (Abb.7). Diese Eisrandposition stimmt gut überein mit der von Reichelt (1960) beobachteten höchsten Lage alpiner Schotter im Gebiet nördlichen von Bad Säckingen, die i.A. als Relikte einer ehemaligen Eisbedeckung durch den Rheingletschers angesehen werden und von ihm mit 420-430 m NN angegeben wurden.

Die Sedimentfüllung des Haselbachtals steht sicherlich nicht in einem unmittelbaren Zusammenhang mit der Entstehung der Randrinne. Wie beschrieben, sind die gut geschichteten Schluffe an der Basis der Sedimentabfolge in den Bohrungen ATU3, 4 und 10 teilweise organisch, was nicht zu einer glazial geprägten Umgebung passt. Viel wahrscheinlicher ist daher, dass der Teil der Randrinne, der das obere Haselbachtal markiert, durch die Sedimentanlieferung des Schöpfebaches aus dem Hotzenwald nach dem Abschmelzen des Gletschers mit grobkörnigen Schottern verfüllt und plombiert wurde und in den tiefer gelegenen, teilweise übertieften Talabschnitten sich Feinsedimente in einem Stillwasserbereich ablagern konnten. Die Grobsedimentverfüllung der Randrinne am Austritt des Schöpfebaches aus dem Schwarzwald wurde erst, nachdem sich der Gewerbebach subsequent in die Felsschwelle nördlich von Bad Säckingen eingeschnitten hatte, teilweise wieder ausgeräumt. Infolgedessen entstand die Wasserscheide zwischen Haselbach und Schöpfebach auf einer $50 \mathrm{~m}$ mächtigen Schotterfüllung.

\subsection{Alter}

Die jüngste Vergletscherung, die das Hochrheintal bei Bad Säckingen betraf, erfolgte während des so genannten Doppelwall-Riß im Rahmen des Riß-Komplexes. Nach GeYER et al. (2003) erstreckte sich der Rheingletscher nach W bis in die Gegend von Bad Säckingen (Abb. 8), ohne jedoch das Möhliner Feld (Abb. 2) zu erreichen, das von verschiedenen Autoren als die Endmoräne des rißzeitlichen Rheingletschers angesehen wird (HantKe 1978, Leser 1987. SChreiner 1995, Verderber 1992, 2003). Stattdessen wird von Müller-Dick (2000) und Tschudi (1904) ein Vorstoß des Schwarzwald-Gletschers aus dem Wehratal (Abb. 2, 8) bis in das südliche Möhliner Feld vorgeschlagen, wo er eine mehrerer Meter mächtige Wallmoräne hinterlassen haben soll. Im Gegensatz dazu nehmen Leser (1987), Schreiner (1995) und Schreiner \& SawatzKI(2000) einen wesentlich schwächeren Vorstoß des Schwarzwaldgletschers über das Wehratal an, der kaum weiter als in den Raum Öflingen nahe der Mündung der Wehra in den Rhein reichte. Eine Konfluenz zwischen Wehratal- und Rheingletscher wird nach LESER (1987) nur für die Zeit maximaler Gletscherausdehnung während der Rißeiszeit angenommen. Als gesichert kann heute gelten, dass der Dinkelberg (Abb. 2, 8) nicht vergletschert war. Stattdessen stieß die Zunge des Wiesegletschers nur bis über Schopfheim hinaus vor (SChreiner 1995, SchreIner \& SaWATZKi 2000). Auch ein Zusammenfluss zwischen Schwarzwald- und Rheingletscher im Bereich des Albtals gilt als widerlegt, nachdem zwischen der Zunge des Albgletschers und dem Rheingletscher Eisrand-Stauseeablagerungen entdeckt wurden (GEYER et al. 2003, WeNDebourg \& Ramshorn 1987). Der genaue Verlauf der rißzeitlichen Eisränder im SüdSchwarzwald und Teilen des Hochrheintals ist bisher nicht bekannt.

Die geomorphologischen Befunde aus dem Bergseegebiet sprechen für eine subglaziale bzw. randglaziale Entstehung des Rinnensystems. Auf randglaziale Rinnen im Bergseegebiet hat bereits ЕRB (1936) hingewiesen. Große Wassermengen waren für die enorme rand- und subglaziale Erosionsleistung erforderlich. Über die 


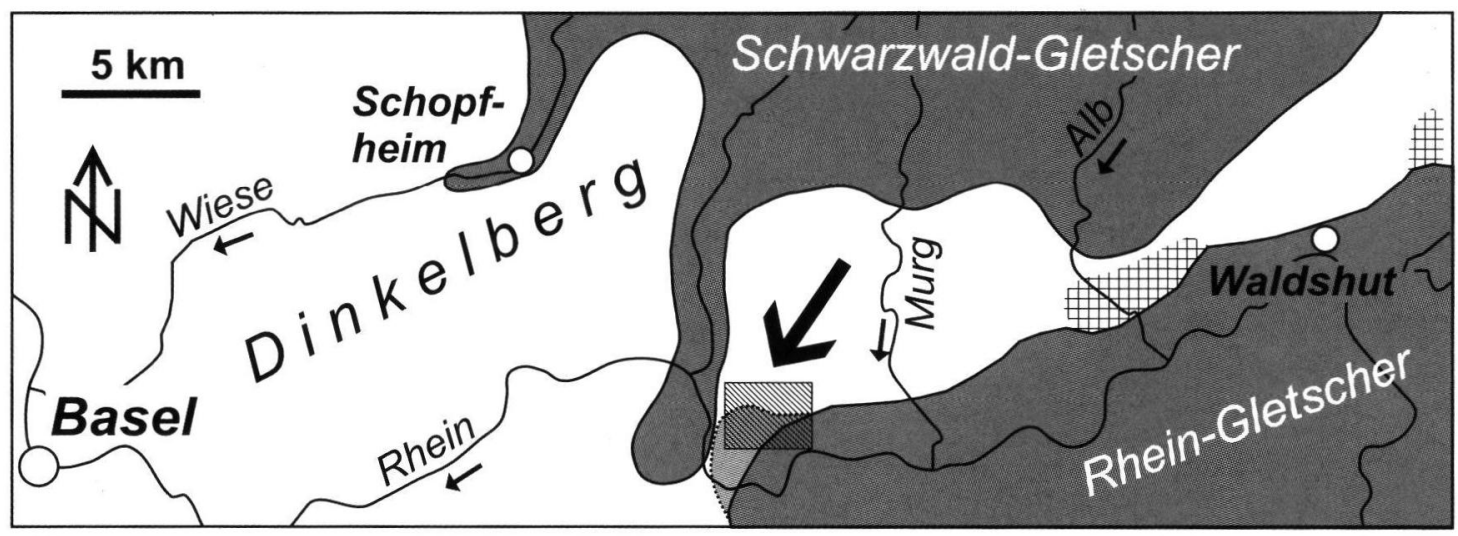

Abb. 8: Maximale Ausdehnung des Schwarzwaldgletschers nach Müller-Dick (2000), Schreiner (1995), Tschudi (1904) und Wendebourg \& Ramshorn (1987) und des Rheingletschers (dunkelgrau) nach GeYer et al. (2003) zurzeit des Doppelwall-Riß. In hellgrau: vermutete Ausdehnung des Rheingletschers während des Doppelwall-Riß aufgrund der Verbreitung subglazialer Felsrinnen. Kreuzschraffiert sind Gebiete ehemaliger Eisstauseen (nach GEYER et al. 2003). Schrägschraffur: Arbeitsgebiet.

Fig. 8: Maximum glacier extend of the Black Forest Glacier after Müller-Dick (2000), SCHreiner (1995), Tschudi (1904) and Wendebourg \& Ramshorn (1987) and the Rhine Glacier (dark grey) after Geyer et al. (2003) during the Doppelwall-Riß. In light grey: supposed extension of the Rhine Glacier based on the distribution of subglacial channels. Cross-hatched areas indicate former ice-dammed lakes (after GEYER et al. 2003). The hatched area is the area of investigation.

Existenz großer Wassermengen am Nordrand des Rheingletschers zurzeit des DoppelwallRiß gibt es keine Zweifel, wie Ablagerungen von Eisrand-Stauseen verschiedentlich belegen (Geyer et al. 2003, Metz 1980, Wendebourg \& RAMSHORN 1987). Der anhand des Rinnensystems von uns im Bergseegebiet definierte Rand des Gletschers fällt sehr gut zusammen mit dem von Erb (1936) und Hantke (1978) vorgeschlagenen rißzeitlichen Gletscherrand und steht in Einklang mit der Verbreitung alpiner Schotter (Reichelt 1960). Die jüngste Alterseinstufung des glazialen Rinnensystems im Bergseegebiet wäre somit doppelwall-rißzeitlich. Die noch deutliche morphologische Ausprägung des Rinnensystems und die teilweise noch wenig verwitterten Felsoberflächen widersprechen dieser Alterseinstufung nicht. Einen direkten Beleg für das Alter der Rinnen gibt es bisher aber nicht.

\section{Zusammenfassung und Ausblick}

Die geomorphologischen und geologischen Untersuchungen auf dem Vorbergsporn des Schwarzwaldes nördlich von Bad Säckingen haben gezeigt, dass die Morphogenese des Tal- und Rinnensystems dieses über $2 \mathrm{~km}$ in das Hochrheintal hineinreichenden Felsriegels wesentlich durch sub- und randglaziale Prozesse geprägt ist. Argumente für eine subglaziale oder randglaziale Entstehung dieser Strukturen wurden genannt. Besonders hervorzuheben ist die Lage der subglazialen Rinnen auf einem Hartgesteins-Felsriegel nahe der Ablationsfront eines ehemaligen Gletschers, die unausgeglichenen Tal-Längsprofile, die charakteristischen Tal-Querprofile, die abrupten Richtungsänderungen der Täler sowie das kaum vorhandene rezente Einzugsgebiet. Da die Geländeformen noch gut zu erkennen sind, wird ihre Entstehung zurzeit des Doppelwall-Riß angenommen, zu dem das Untersuchungsgebiet im di- 
rekten Einflussbereich des Rheingletschers und auch des Schwarzwaldgletschers lag.

Verschiedene Fragen konnten bisher nicht geklärt werden. Beispielsweise wird die Fortsetzung des subglazialen Rinnensystems südlich des Bergsees durch örtlich recht mächtige Löss- und Lösslehmvorkommen verdeckt. Der Verlauf im Untergrund vorhandener Rinnen könnte dort evtl. mit Hilfe geophysikalischer Methoden kartiert werden. Eine genauere Kenntnis des Verlaufs der Felssohle und der Sedimentfüllung in den gut sichtbaren Rinnen wäre ebenfalls zur Überprüfung der vorgeschlagenen Morphogenese hilfreich. Geophysikalische Methoden in den engen und z.T. recht tiefen Tälern sind hier aber wenig zweckmäßig. Bohrungen an geeigneten Stellen könnten Sedimente liefern, die viele organische Bestandteile enthalten und mit deren Hilfe eine zeitliche Einstufung der Sedimentfüllungen verschiedener Täler beispielsweise palynostratigraphisch vorgenommen werden kann. Schließlich wäre auch die Möglichkeit zu prüfen, ob die Rinnen im Bergseegebiet nicht direkt mit Hilfe von Expositionsaltersbestimmungen (z.B. Beryllium-Methode) datierbar sind. Damit könnte die vorgeschlagene Alterseinstufung verifiziert (oder widerlegt) werden. Vielleicht ergäben sich so auch wichtige Anhaltspunkte zur zeitlichen Einordnung des Doppelwall-Riß.

\section{Dank}

Die Untersuchungen im Bergsee und seiner Umgebung wurden im Rahmen mehrerer Projekte zur Erforschung der prähistorischen Seismizität in der Baselregion durch den SNF, die ETH Zürich sowie die Universität Zürich gefördert. Unser Dank gilt besonders den Herren Professoren D. Giardini, Institut für Geophysik, ETH Zürich, und W. Haeberli, Geographisches Institut, Universität Zürich, für ihr großes Interesse am Fortgang der Un- tersuchungen und für zahlreiche Diskussionen. Der Stadt Bad Säckingen, vertreten durch den ehemaligen Bürgermeister Herrn Dr. Dr. Nufer sowie dem Umweltbeauftragten Herrn R. Däubler, danken wir für das Einverständnis zu den Geländearbeiten und ihr reges Interesse am Fortgang der Untersuchungen an ,ihrem“ See. Schließlich gilt unser Dank Herrn J. Turtur von der Schluchseewerke AG für die Erlaubnis, noch nicht veröffentlichte Bohrdaten aus dem Haselbachtal publizieren zu dürfen.

\section{Schriftenverzeichnis}

Angelstein, S. (2002): Geomorphologische Kartierung: Bergsee und Umgebung unter Berücksichtigung der vermuteten glazialen Tunneltäler. - Ber. Berufspraktikum ETHZ u. UNIZ, 10 S.; Zürich.

BECKer, A. (2003): Vom Denekamp-Interstadial zur Gegenwart - 30‘000 Jahre Erdgeschichte überliefert in den Sedimenten des Bergsees bei Bad Säckingen. - Regio Basiliensis, 44 (3): 261-273; Basel.

Becker, A., Bucher, F., Davenport, C.A. \& Fusch, A. (2004): Geotechnical characteristics of post-glacial organic sediments in Lake Bergsee, southern Black Forest, Germany. - Eng. Geol., 74: 91-102; Amsterdam.

Becker, A., Davenport, C.A. \& Giardini, D. (2002): Palaeoseismicity studies on end-Pleistocene and Holocene lake deposits around Basle, Switzerland. - Geophys. J. Int., 149: 659-678; Oxford.

Beising, E. (2003): Pleistozäne Sedimente am Südrand des Hotzenwaldes. - Regio Basiliensis, 44 (3): 251-260; Basel.

Benn, D.I. \& Evans, D.J.A. (1998): Glaciers and glaciation. - 734 S.; London (Arnold).

Booth, D.B. \& Hallet, B. (1993): Channel networks carved by subglacial water: Observations and reconstructions in the 
eastern Puget Lowland of Washington.Bull. Geol. Soc. Am., 105: 671-683; Boulder.

DAHL, R. (1965): Plastically sculptured detail forms on rock surfaces in northern Nordland, Norway. - Geografiska Annaler, 47 A: 83-140; Stockholm.

EMbleton, C. \& KING, C.A.M. (1975): Glacial geomorphology. 1. - 573 S.; London (Arnold).

ERB, L. (1936): Zur Stratigraphie des mittleren und jüngeren Diluviums in Südwestdeutschland und dem angrenzenden schweizerischen Grenzgebiet. - Mitt. Bad. Geol. L.-A., 11: 187-220; Freiburg i. Br.

Geyer, O.F., Schober, T. \& Geyer, M. (2003): Die Hochrhein-Region zwischen Bodensee und Basel. - Sammlung geol. Führer, 94: 526 S.; Berlin, Stuttgart (Borntraeger).

Haldimann, P., Naef, H. \& Schmassmann, H. (1984): Fluviatile Erosions- und Akkumulationsformen als Indizien jungpleistozäner und holozäner Bewegungen in der Nordschweiz und angrenzenden Gebieten. - NAGRA Techn. Ber., 84-16: 112 S; Baden.

HantKe, R. (1978): Eiszeitalter. 1: Die jüngste Erdgeschichte der Schweiz und ihrer Nachbargebiete. - 468 S.; Thun (Ott).

LESER, H. (1987): Zur Glazialproblematik auf Blatt Freiburg-Süd der Geomorphologischen Karte 1 : 100000 der Bundesrepublik Deutschland (GMK 100, Blatt 2).Eiszeitalter und Gegenwart, 37: 139-144; Hannover.

Menzies, J. (1996): Past glacial environments: Sediments, forms and techniques. - Glacial Environments, 2: 598 S.; $\quad$ Oxford (Butterwort-Heinemann).

Metz, R. (1980): Geologische Landeskunde des Hotzenwaldes. - 1117 S.; Lahr (Schauenburg).

MüLleR, P.C. (1993): Die Wuhren. Ein Beitrag zur Wirtschaftsgeschichte des Hochrheins und des Hotzenwaldes. - Beitr. Volkskunde Bad.-Württ., 5: 333-350; Freiburg i.Br., Stuttgart.

MüLleR-DicK, K.A. (2000): Möhliner Feld im Hochrheintal. - In: Kelly, M., Linden, U. \& Schlüchter, C. (Hrsg.): DEUQUA 2000, Bern, Exkursionsführer, Exk. A1: 1013; Hannover.

ReicheLt, G. (1960): Quartäre Erscheinungen im Hotzenwald zwischen Wehra und Alb. - Ber. Naturf. Ges. Freiburg i. Br., 50: 57127; Freiburg i. Br..

SawatzKi, G. (1997): Geologische Karte von Baden-Württemberg 1:25000, vorläufige Ausgabe, 8413 Bad Säckingen. - 32 S., Freiburg i. Br. (Geol. L.-Amt).

Schreiner, A. (1995): Zur Quartärgeologie des unteren Wehratals und zur Frage der Vergletscherung des Dinkelberges in der Rißeiszeit (SW Deutschland). - Eiszeitalter u. Gegenwart, 45: 62-71; Hannover.

Schreiner, A. \& Sawatzki, G. (2000): Der Wiesetalgletscher im Südschwarzwald in der Würm- und Rißeiszeit. - Jber. Mitt. oberrhein. geol. Ver., N.F. 82: 377-410; Stuttgart.

Sugden, D.E. \& John, B.S. (1976): Glaciers and landscape: A geomorphological approach. - 376 S.; London (Arnold).

Trefzger, E. (1965): Quartäre Hebung des Hotzenwaldes.- Jber. Mitt. oberrhein. geol. Ver., N.F. 47: 45-60; Stuttgart.

Tschudi, R. (1904): Zur Altersbestimmung der Moränen im unteren Wehratal. - Diss. Univ. Basel: 29 S.; Basel.

VERDERBER, R. (1992): Quartärgeologische Untersuchungen im Hochrheingebiet zwischen Schaffhausen und Basel. - Diss. Univ. Freiburg i.Br.: 183 S.; Freiburg i. Br.

Verderber, R. (2003): Quartärgeologie im Hochrheingebiet zwischen Schaffhausen und Basel. - Z. dt. geol. Ges., 154 (2-3): 369-406; Stuttgart.

Wendebourg, G.M. \& Ramshorn, C. 
(1987): Der Verzahnungsbereich alpiner Wüthrich, C. (2003): Der Bergsee Bad und südostschwarzwälder Rißvereisung Säckingen: Die Revitalisierung eines (Baden-Württemberg). - Jh. Geol. L.-A. urbanen Sees. - Regio Basiliensis, 44 (3): Bad.-Württ., 29: 255-268; Freiburg i.Br. 205-220; Basel. 